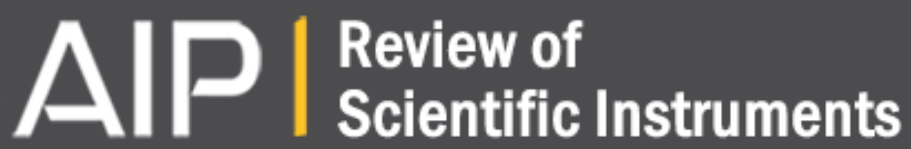

Measurements of poloidal rotation velocity using cross-correlation spectroscopy in the $\mathrm{H}-1$ heliac

M. G. Shats, H. Punzmann, H. Xia, and W. M. Solomon

Citation: Review of Scientific Instruments 74, 2044 (2003); doi: 10.1063/1.1537032

View online: http://dx.doi.org/10.1063/1.1537032

View Table of Contents: http://scitation.aip.org/content/aip/journal/rsi/74/3?ver=pdfcov

Published by the AIP Publishing

\section{Articles you may be interested in}

Gyrokinetic turbulent transport simulation of a high ion temperature plasma in large helical device experiment Phys. Plasmas 19, 042504 (2012); 10.1063/1.4704568

Ultrafast spectroscopy diagnostic to measure localized ion temperature and toroidal velocity fluctuationsa) Rev. Sci. Instrum. 81, 10D714 (2010); 10.1063/1.3479114

Study of intermittent small-scale turbulence in Wendelstein 7-AS plasmas during controlled confinement transitions

Phys. Plasmas 12, 012507 (2005); 10.1063/1.1818142

A multi-channel spectroscopic system for measuring impurity ion temperatures and poloidal rotation velocities in TJ-II

Rev. Sci. Instrum. 72, 971 (2001); 10.1063/1.1326008

Measurements of poloidal rotation velocity using charge exchange spectroscopy in a large helical device Rev. Sci. Instrum. 71, 2360 (2000); 10.1063/1.1150621

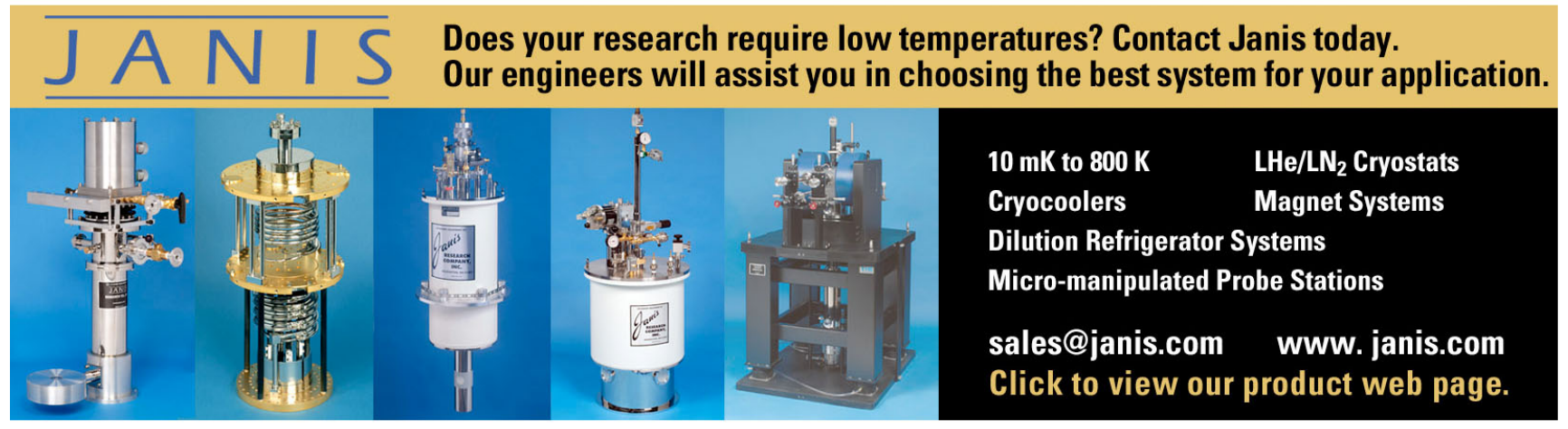




\title{
Measurements of poloidal rotation velocity using cross-correlation spectroscopy in the $\mathrm{H}-1$ heliac
}

\author{
M. G. Shats, ${ }^{\text {a) }}$ H. Punzmann, H. Xia, and W. M. Solomon \\ The Australian National University, Canberra, Australia
}

(Presented on 10 July 2002)

\begin{abstract}
A correlation spectroscopy diagnostic [M.G. Shats and J. Howard, Fusion Eng. Des. 34-35, 271 (1997)] measures fluctuation spectra and local fluctuation intensities in a radiation-dominated plasma, such as the low-temperature plasma in the H-1 heliac $\left(T_{e}<50 \mathrm{eV}, n_{e}<2 \times 10^{18} \mathrm{~m}^{-3}\right)$. When the fluctuation coherence lengths in the poloidal and radial directions are shorter than the plasma radius, the cross-correlation function of the two crossed-sightline fluctuating intensities contains information about the fluctuations amplitude and their phase in the intersection volume. The optical setup on the $\mathrm{H}-1$ heliac uses two nearly orthogonal views to image 20 optical fibers arranged into two linear arrays in the plasma poloidal cross section. A matrix of $10 \times 10$ cross-correlation functions is then analyzed to determine the poloidal phase velocity of the fluctuations, poloidal and radial correlation lengths, and the radial profiles of the fluctuations intensity. The results on the poloidal propagation velocity measured using the cross-correlation technique (time delay of the cross-correlation functions) are compared with the poloidal velocity measured using poloidally separated probes in the plasma. Both velocities are found to be in good agreement and also agree well with the $\mathbf{E} \times \mathbf{B}$ drift velocity in this plasma. (C) 2003 American
\end{abstract} Institute of Physics. [DOI: 10.1063/1.1537032]

The radial electric-field $E_{r}$, measurements are crucial for understanding plasma transport and confinement. ${ }^{1}$ Such measurements are difficult whether one uses the heavy ionbeam probe technique to measure the electrostatic plasma potential or the charge-exchange spectroscopy to derive $E_{r}$ using the zero-order radial force balance. In the latter case, the accuracy in the $E_{r}$ estimate depends on the quality of the data on the ion pressure gradients, and the ion flow velocities. In the presence of turbulence, the $\mathbf{E} \times \mathbf{B}$ drift velocity often dominates the fluctuation propagation velocity in the lab frame. By measuring the poloidal velocity of the turbulence it is possible in some cases to reliably estimate $E_{r}$.

In this article, the results on the poloidal rotation velocity measured using the cross-correlation analysis of the twoview, ten-channel visible spectroscopy diagnostic on the $\mathrm{H}-1$ toroidal heliac are presented. The cross-correlation technique has been used for the turbulence characterization using different diagnostics, such as the correlation electron cyclotron emission (ECE), ${ }^{2}$ beam emission spectroscopy, ${ }^{3}$ soft x-ray signal analysis, ${ }^{4}$ correlation spectroscopy, ${ }^{5}$ etc. The technique relies on the ensemble averaging of the uncorrelated fluctuations. Recently, an extended version of the method has been proposed ${ }^{6}$ which uses four lines of sight lying in two parallel planes. Such an extension allows another dimension (e.g., toroidal) in the turbulence characterization to be introduced.

Now we discuss the principles of the cross-correlation technique with regard to the turbulence propagation using the cell model terminology, as in Ref. 6. The plasma volume consists of square (or cubic) cells such that the light intensity

${ }^{a)}$ Electronic mail: michael.shats@anu.edu.au fluctuations are completely correlated inside each cell and the correlation decreases as a function of the distance between the cells. In the model, ${ }^{6}$ it is assumed that the crosscorrelation between fluctuations originating from any two different cells is zero, or, in other words, the correlation length is of the order of the viewing chord diameter. This is not the case in the $\mathrm{H}-1$ heliac, where turbulence is often dominated by large coherent structures. ${ }^{5}$ Figure 1(a) shows three viewing chords in the plasma poloidal cross section. Solid lines represent the heliac flux surfaces and squares along each of the viewing chords correspond to the local cells. The fluctuating intensity of light collected along vertical and horizontal chords is a sum of the local fluctuating intensities $\widetilde{\varepsilon}_{i}(t)$ and $\widetilde{\sigma}_{i}(t): \widetilde{I}_{1}=\Sigma_{i} \widetilde{\varepsilon}_{i}$ and $\widetilde{I}_{2}=\Sigma_{j} \widetilde{\sigma}_{j}$. The cross-correlation function between two chord-average intensities is determined as

$$
\left\langle\widetilde{I}_{1} \widetilde{I}_{2}\right\rangle=\sum_{i, j}\left\langle\widetilde{\varepsilon}_{i} \widetilde{\sigma}_{j}\right\rangle=\left\langle\widetilde{\varepsilon}_{k} \widetilde{\sigma}_{k}\right\rangle+\sum_{i \neq j}\left\langle\widetilde{\varepsilon}_{i} \widetilde{\sigma}_{j}\right\rangle .
$$

When the turbulence correlation length $l_{c}$ is of the order of the distance between the cells, the correlation (1) is determined only by the cross-correlation $\left\langle\widetilde{\varepsilon}_{k} \widetilde{\sigma}_{k}\right\rangle$ from the cell representing the intersection between two viewing chords. Here, by the cross correlation we mean the cross-correlation function, $\mathrm{CF}(\tau)$, determined as

$$
\mathrm{CF}(\tau)=\langle\widetilde{\varepsilon}(t) \widetilde{\sigma}(t)\rangle=\frac{\langle\widetilde{\varepsilon}(t) \widetilde{\sigma}(t+\tau)\rangle}{\sqrt{\langle\widetilde{\varepsilon}(t)\rangle^{2}\langle\widetilde{\sigma}(t+\tau)\rangle^{2}}},
$$

where the time lag $\tau$ between the signals $\widetilde{\varepsilon}_{i}(t)$ and $\widetilde{\sigma}_{i}(t)$. If fluctuating signals are dominated by coherent oscillations, usually coexisting with the broadband turbulence, and this is the case in the described experiments, the cross-correlation 

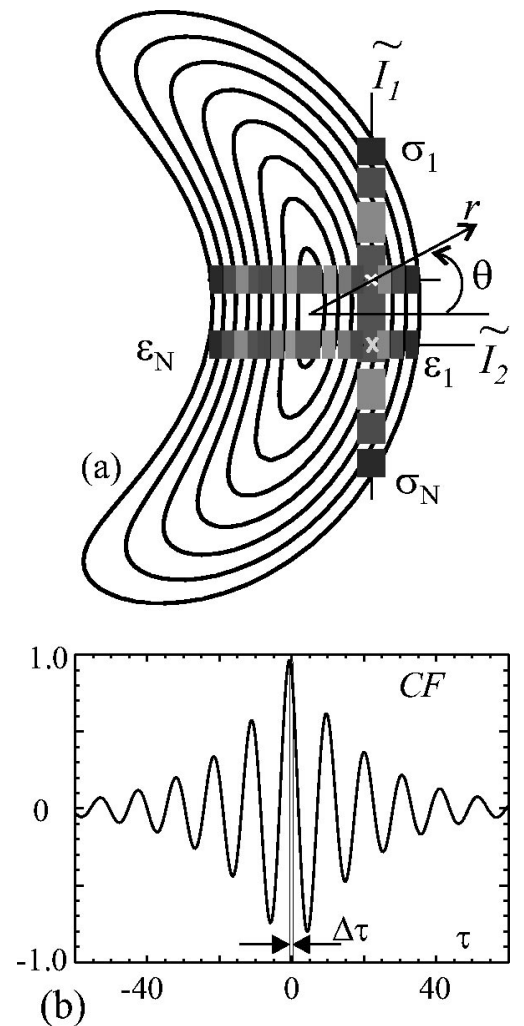

FIG. 1. (a) Schematic of the collection geometry and (b) an example of the cross-correlation function.

function will look like a decaying periodic function of the time delay $\tau$, depicted in Fig. 1(b). This function has a maximum at $\tau=\Delta \tau$ and its amplitude is $\operatorname{CF}(\tau=\Delta \tau) \leqslant 1$. When $\widetilde{\varepsilon}_{i}(t)=\widetilde{\sigma}_{i}(t)$, the time delay $\Delta \tau=0$ and $\operatorname{CF}(\tau=0)=1$. The maximum of the cross-correlation function for two cells separated radially by $\Delta r$ and poloidally by $\Delta \theta$ (where $\theta$ is the poloidal angle) will be less than unity for any finite radial $l_{\text {rad }}$, and poloidal $l_{\theta}$, correlation lengths in the plasma

$$
\begin{aligned}
\operatorname{Max}\left[\left\langle\widetilde{\varepsilon}_{i} \widetilde{\sigma}_{j}\right\rangle\right] & =\operatorname{CF}(\Delta r, \Delta \theta, \tau=\Delta \tau) \\
& <\exp \left\{-\left|\frac{\Delta r}{l_{\mathrm{rad}}}\right|-\left|\frac{\Delta \theta}{l_{\theta}}\right|\right\} .
\end{aligned}
$$

Modeling shows that in the case of the propagating turbulence, when $\Delta \tau_{k k} \neq \Delta \tau_{i j}$, and for long but finite correlation lengths, $l_{\theta} \sim 2 r$ and $l_{\mathrm{rad}} \sim 0.5 r$ (where $r$ is the plasma radius), $\operatorname{Max}\left[\left\langle\widetilde{\varepsilon}_{k} \widetilde{\sigma}_{k}\right\rangle\right]>\operatorname{Max}\left[\left\langle\widetilde{\varepsilon}_{i} \widetilde{\sigma}_{j}\right\rangle_{i \neq j}\right]$, and the maximum of the cross-correlation function of the chord-averaged intensities Eq. (1) coincides in time with the maximum of the crosscorrelation function originating from the intersection volume: $\Delta \tau\left(\left\langle\widetilde{I}_{1} \widetilde{I}_{2}\right\rangle\right) \approx \Delta \tau\left(\left\langle\widetilde{\varepsilon}_{k} \widetilde{\sigma}_{k}\right\rangle\right)$. An example of such a computation is shown in Fig. 2, where the individual contributions $\left\langle\widetilde{\varepsilon}_{i} \widetilde{\sigma}_{j}\right\rangle$ [Fig. 2(a)] are summed to model the finite correlation length effect $\Sigma\left\langle\widetilde{\varepsilon}_{i} \widetilde{\sigma}_{j}\right\rangle$ [Fig. 2(b)].

Thus, even if the turbulence correlation lengths in the plasma are long (e.g., of the order of the plasma radius), it is possible to determine the local fluctuation phase and the phase velocity. If we choose a pair of chord-average signals [e.g., $\widetilde{I}_{1}$ and $\widetilde{I}_{2}$ as shown in Fig. 1(a)] characterized by the intersection point $\left(r_{0}, \theta_{0}\right)$ and whose $\mathrm{CF}$ has maximum at $\tau=\Delta \tau_{0}$ and another pair characterized by $\left(r_{0}, \theta_{1}\right)$ and $\tau$

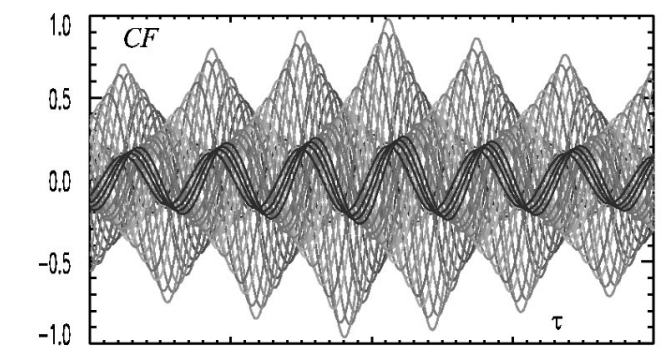

(a)

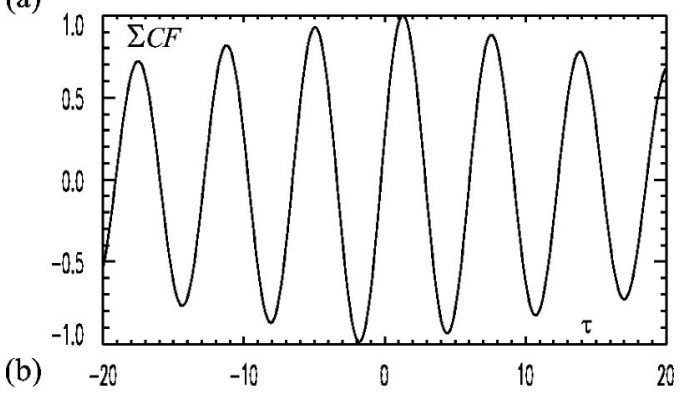

FIG. 2. (a) Cross-correlation functions originating from 34 poloidally separated cells around the chord intersection cell $C_{0}$ are time-delayed proportionally to the distance away from $C_{0}$ and (b) the normalized sum of these cross-correlation functions. The poloidal correlation length in the model equals to two plasma radii.

$=\Delta \tau_{1}$, then we associate a time delay $d \tau=\Delta \tau_{1}-\Delta \tau_{0}$ with the propagation velocity in the poloidal direction. Below, we discuss the implementation of this idea in the H-1 stellarator.

Experiments have been performed in the $\mathrm{H}-1$ helical axis stellarator ${ }^{7}$ having a major radius of $R_{0}=1.0 \mathrm{~m}$ and a minor plasma radius of less than $0.2 \mathrm{~m}$. In the experiments discussed here, $\mathrm{H}-1$ was operated at low-magnetic fields $(<0.2 \mathrm{~T})$ with the current-free plasma produced by the pulsed radio-frequency (rf) power of less than $100 \mathrm{~kW}$ at 7 MHz. Plasma conditions were as follows (for more details see, e.g., Ref. 8): the electron density of the argon discharges is $n_{e}=(0.5-1.0) \times 10^{18} \mathrm{~m}^{-3}$, the electron temperature $T_{e}$ $=(5-40) \mathrm{eV}$ and the ion temperature is $T_{i}=(20-60) \mathrm{eV}$. In these radiation-dominated discharges strong fluctuations in the electrostatic potential, electron temperature, and density are observed. The poloidal propagation velocity of these fluctuations is estimated using the cross-correlation spectroscopy diagnostic and results are compared with the measurements of the poloidal velocity from the electrostatic probes.

The optical setup is similar to that described in Ref. 9 but the light is collected from two orthogonal views ten chords in each as shown in Fig. 3. Chords represent images in the plasma of the $2 \times 10$-channel optical fiber arrays. These arrays $(10 \times 1 \mathrm{~mm}$ core diameter fibers $)$ are imaged in the plasma using two camera zoom lenses ( $f$ $=35-105 \mathrm{~mm}$ ). The geometry in Fig. 3 corresponds to $f$ $=70 \mathrm{~mm}$ that results in the magnifications of $m_{y}=13.8$ and $m_{x}=9.6$ in the vertical (in fact, tangential to the flux surface) and horizontal views correspondingly. The grid produced by the two views of lines of sight has a cell with sides of approximately $\Delta_{\mathrm{rad}}=16.5 \mathrm{~mm}$ and $\Delta_{\text {pol }}=15.9 \mathrm{~mm}$ in the radial and poloidal directions correspondingly. Cross-correlation functions $(\mathrm{CCF})$ are computed as 


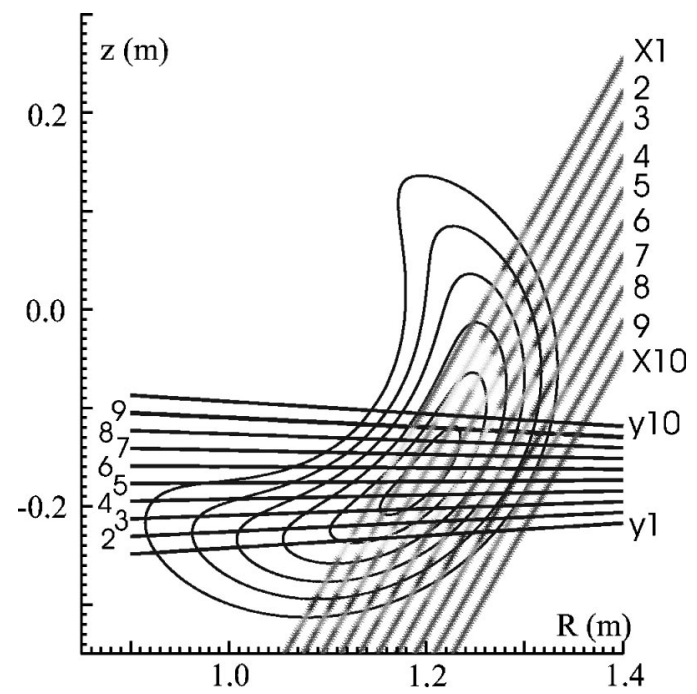

FIG. 3. Viewing chords geometry in the $\mathrm{H}-1$ heliac.

$$
\begin{aligned}
\operatorname{CCF} & \left(x_{i} y_{j}, \tau\right) \\
& =\left\langle\widetilde{I}\left(x_{i}, t\right) \widetilde{I}\left(y_{j}, t+\tau\right)\right\rangle /\left\{\sqrt{\left\langle\widetilde{I}\left(x_{i}, t\right)^{2}\left\langle\widetilde{I}\left(y_{j}, t+\tau\right)^{2}\right\rangle\right\rangle}\right\},
\end{aligned}
$$

where the time lag $\tau$ between the signals $x_{i}$ and $y_{j}$ is in the range $\tau=(-T / 2, T / 2)$ and $T$ is the analyzed time interval. For the results presented in the article, signals are digitized at the rate of $2 \mathrm{MHz}$ and $T$ is typically $T=10 \mathrm{~ms}$. CCFs are computed for intersecting "vertical" and "horizontal" chords to produce a matrix of $10 \times 10=100 \mathrm{CCFs}, 20000$ elements in each.

Figure 4 shows a frequency power spectrum of the chord-average line radiation at $\lambda=488 \mathrm{~nm}$ (ArII) measured along the horizontal chord $y 4, S_{y 4}(f)=\sqrt{P_{y 4}^{*}(f) P_{y 4}(f)}$, [Fig. 4(a)] and the cross-power spectrum between chords $x 7$ and $y 4, S_{x 7 y 4}(f)=P_{x 7}^{*}(f) P_{y 4}(f)$, [Fig. 4 (b)]. Here, $P_{y n}$ denotes the Fourier transform of the signal $y_{n}$ and asterisk de-
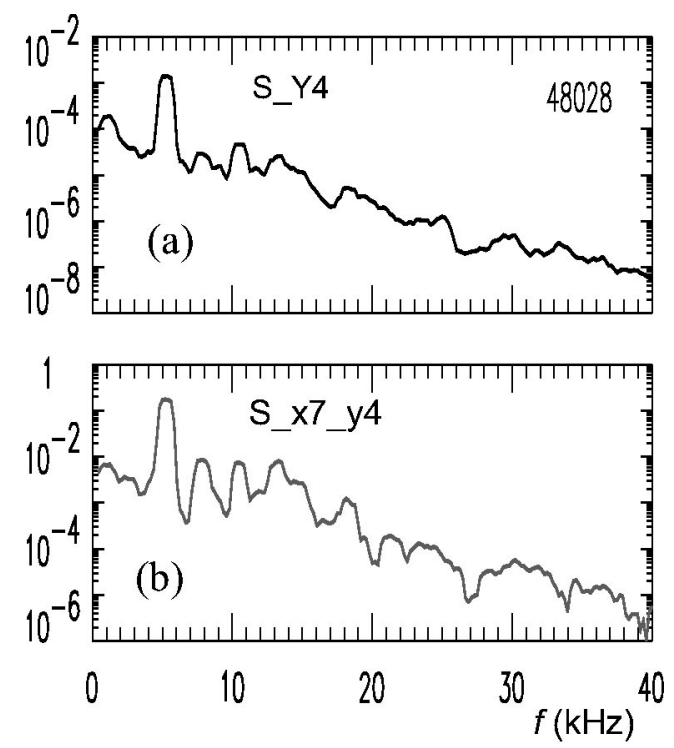

FIG. 4. Frequency spectra of the (a) fluctuations in the spectral line intensity collected along the horizontal chord $y 4$ and (b) the cross-power spectrum of the signals $x 7$ and $y 4$.

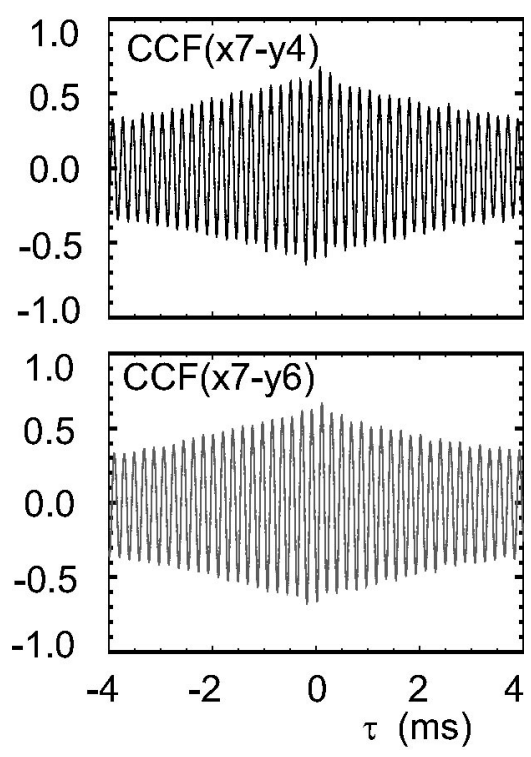

FIG. 5. Cross-correlation functions between signals (a) $x 7-y 4$ and (b) $x 7-y 6$.

notes the complex conjugate. As it has been previously observed using probes, the fluctuation spectra show both the broadband exponentially decaying turbulence and several coherent modes in the range of $f=3-20 \mathrm{kHz}$.

Figure 5 shows CCFs computed using two poloidally separated signals $y_{4}$ and $y_{6}$ "crossed" with the mid radius vertical chord signal $x_{7}$. Since the signal spectra are dominated by a strong coherent mode at the frequency of about 6 $\mathrm{kHz}$ (seen in Fig. 4) the cross-correlation functions are relatively slow-decaying sinusoidal functions. The correlation time is typically about $6 \mathrm{~ms}$. Nevertheless, a well-defined maximum of the CCF is always observed. A time delay at which the CCFs are peaked is nonzero and it is used here to estimate the propagation velocity of the fluctuations. The delay between the two maxima of the CCFs shown in Fig. 5 is between 1 and $30 \mu$ s. Figure 6 shows the delay between the CCFs maxima as a function of the separation $\Delta y$ between the horizontal chords $y_{4}$ to $y_{7}$ cross-correlated with $x_{5}$. The time delay is a linear function of $\Delta y$ for the

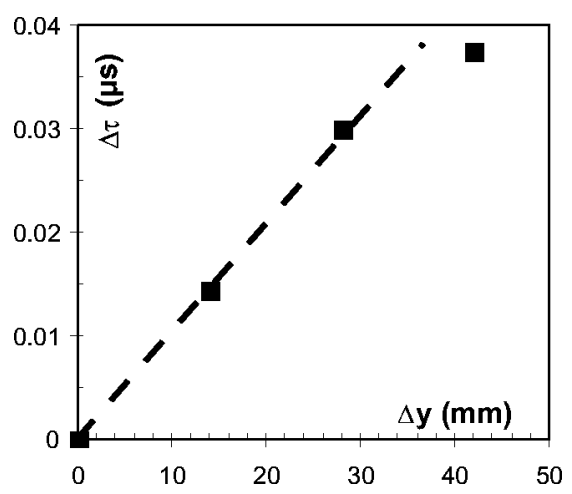

FIG. 6. Time delay $\Delta \tau$ between maxima of the pairs of the cross-correlation functions as a function of the poloidal separation $\Delta y$ between horizontal viewing chords. The following pairs were used: $\operatorname{CCF}\left(x_{5}-y_{4}\right)-\operatorname{CCF}\left(x_{5}-y_{5}\right)$, $\mathrm{CCF}\left(x_{5}-y_{5}\right)-\mathrm{CCF}\left(x_{5}-y_{6}\right), \quad \operatorname{CCF}\left(x_{5}-y_{4}\right)-\mathrm{CCF}\left(x_{5}-y_{6}\right), \quad \operatorname{CCF}\left(x_{5}-y_{5}\right)$ $-\mathrm{CCF}\left(x_{5}-y_{7}\right), \operatorname{CCF}\left(x_{5}-y_{4}\right)-\mathrm{CCF}\left(x_{5}-y_{7}\right)$. 


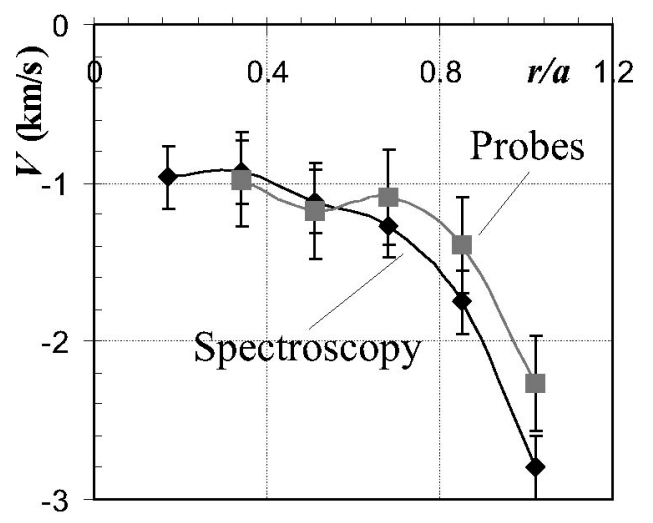

FIG. 7. Radial profiles of the turbulence poloidal propagation velocity as measured using probes (squares) and derived from the time delay spectroscopic technique (diamonds).

pairs of CCF separated by up to two chords, such as $\quad \operatorname{CCF}\left(x_{5}-y_{4}\right)-\mathrm{CCF}\left(x_{5}-y_{5}\right), \operatorname{CCF}\left(x_{5}-y_{5}\right)-\operatorname{CCF}\left(x_{5}-y_{6}\right)$, $\operatorname{CCF}\left(x_{5}-y_{4}\right)-\operatorname{CCF}\left(x_{5}-y_{6}\right), \operatorname{CCF}\left(x_{5}-y_{5}\right)-\operatorname{CCF}\left(x_{5}-y_{7}\right)$. However, for larger poloidal separation, e.g., $\operatorname{CCF}\left(x_{5}-y_{4}\right)-\operatorname{CCF}\left(x_{5}-y_{7}\right), \Delta \tau$ deviates from the straight line, probably because the $x_{5}-y_{4}$ and $x_{5}-y_{7}$ intersection points do not lie on the same flux surface.

The poloidal propagation velocity of the electrostatic potential fluctuations has also been measured using the floating potentials from two poloidally separated probes $(\Delta y$ $=3.5 \mathrm{~mm})$. The phase between them $\Delta \varphi$ is computed in the frequency domain and the poloidal wave number is computed as $k_{\mathrm{pol}}(\omega)=\Delta \varphi(\omega) / \Delta y .{ }^{8}$ The poloidal propagation velocity derived from the probes is determined as $V$ $=\omega / k_{\text {pol }}$ and it agrees with the measured $\mathbf{E} \times \mathbf{B}$ drift velocity, $V_{\mathrm{E}}=E_{r} / B_{T}$ to within $10 \%$.
Figure 7 shows the comparison between the poloidal propagation velocities of the fluctuations as determined using the cross-correlation analysis of the line intensities ( $V_{\mathrm{pol}}$ $=\Delta y / \Delta \tau)$ and the probes. The radial profile of the velocity from the cross-correlation technique is obtained by measuring the time delays between the maxima of the $\operatorname{CCF}\left(x_{4}-y_{4}-y_{6}\right), \ldots, \operatorname{CCF}\left(x_{9}-y_{4}-y_{6}\right)$. A reasonable agreement between the two poloidal velocities is observed over a wide radial region.

Summarizing, it has been demonstrated that using a relatively simple experimental setup a good estimate of the plasma poloidal rotation velocity has been obtained from the chord-average spectroscopic measurements of the line emission. This technique has also been used to derive the poloidal rotation velocities in the electron cyclotron resonance heated (ECRH) plasma at higher-magnetic field $(B=0.5 \mathrm{~T})$. In this case, the broadband turbulence dominates the turbulence spectra. The time-delay analysis has produced a plausible estimate of the poloidal drift velocity, though the time resolution has not always been sufficient due to the higher velocities observed in the ECRH plasma. A higher digitization rate (up to $4-6 \mathrm{MHz}$ ) is necessary to improve the resolution of the method.

${ }^{1}$ K. Itoh and S. I. Itoh, Plasma Phys. Controlled Fusion 38, 1 (1996).

${ }^{2}$ C. E. Thomas, Jr. and R. F. Gandy, Rev. Sci. Instrum. 61, 3570 (1990).

${ }^{3}$ R. D. Durst, R. J. Fonck, G. Cosby, H. Evensen, S. F. Paul, Rev. Sci. Instrum. 63, 4907 (1992).

${ }^{4}$ J. Karlsson, I. Pazsit, and R. D. Gill, Fusion Eng. Des. 34-35, 175 (1997).

${ }^{5}$ M. G. Shats and J. Howard, Fusion Eng. Des. 34-35, 271 (1997).

${ }^{6}$ A. Ejiri, Rev. Sci. Instrum. 73, 1766 (2002).

${ }^{7}$ S. M. Hamberger, B. D. Blackwell, L. E. Sharp, and D. B. Shenton, Fusion Technol. 17, 123 (1990).

${ }^{8}$ M. G. Shats, Plasma Phys. Controlled Fusion 41, 1357 (1999).

${ }^{9}$ H. Punzmann, M. G. Shats, W. M. Solomon, and H. Xia, Rev. Sci. Instrum. (these proceedings). 